\title{
Outcomes of abdominal esophageal cancer patients who were treated with esophagectomy
}

\author{
HIROYUKI KITAGAWA ${ }^{1}$, TSUTOMU NAMIKAWA ${ }^{1}$, JUN IWABU ${ }^{1}$, KAZUNE FUJISAWA ${ }^{1}$, \\ MICHIYA KOBAYASHI $^{2}$ and KAZUHIRO HANAZAKI ${ }^{1}$
}

\author{
Departments of ${ }^{1}$ Surgery and ${ }^{2}$ Human Health and Medical Sciences, Kochi Medical School, Nankoku, Kochi 783-8505, Japan
}

Received June 14, 2017; Accepted October 2, 2017

DOI: $10.3892 / \mathrm{mco} .2017 .1510$

\begin{abstract}
The aim of the present study was to elucidate the characteristics and outcomes of abdominal esophageal cancer patients who were treated with esophagectomy. The records of 210 esophagectomy patients were retrospectively reviewed and the differences in postoperative outcomes and disease-specific survival between squamous cell carcinoma and adenocarcinoma were evaluated. Of the 20 abdominal esophageal cancer patients, 11 had squamous cell carcinoma, 8 had adenocarcinoma and 1 had adenosquamous cell carcinoma. The body mass index and serum albumin levels were significantly lower in the squamous cell carcinoma patients compared with those in the adenocarcinoma patients, and abdominal lymph node metastasis was significantly more frequent in the adenocarcinoma patients. Early recurrence occurred in 5 patients who had postoperative surgical site infection, microscopic residual cancer, and mediastinal lymph node metastasis. A Kaplan-Meier curve indicated a significantly shorter survival time in patients who underwent surgery with a thoraco-abdominal approach, who had postoperative complications, and who had microscopic residual cancer. This study demonstrated the significance of $\mathrm{R} 0$ resection and prevention of postoperative complications in improving the prognosis of patients with abdominal esophageal cancer.
\end{abstract}

\section{Introduction}

Esophageal squamous cell carcinoma (SCC) of the thoracic esophagus is common in Asia (1). In Japan, $>85 \%$ of esophageal carcinoma cases are located in the thoracic part of the esophagus (2), and preoperative chemotherapy followed by radical esophagectomy with three-field lymphadenectomy is considered to be the standard treatment (3). In Western

Correspondence to: Dr Tsutomu Namikawa, Department of Surgery, Kochi Medical School, 185-1 Kohasu-Okocho, Nankoku, Kochi 783-8505, Japan

E-mail: tsutomun@kochi-u.ac.jp

Key words: abdominal esophageal cancer, esophagectomy, postoperative complications, microscopic residual cancer countries, adenocarcinoma (adenoCa) of the esophago-gastric junction (EGJ) is common (4), and is mainly treated by preoperative chemoradiotherapy followed by surgery $(5,6)$.

Siewert et al classified EGJ carcinoma into three types (7), namely type I (abdominal esophageal carcinoma), type II (true carcinoma of the cardia), and type III (gastric cancer). A Japanese randomized controlled study recommended an abdominal approach rather than a left thoraco-abdominal approach (TAA) for patients with type II and III EGJ adenoCa (8). However, there have been few reports regarding the optimal treatment method for patients with abdominal esophageal cancer (AEC), including SCC (Siewert type I cancer).

The aim of the present study was to elucidate the characteristics and outcomes of AEC patients who were treated with esophagectomy.

\section{Patients and methods}

Patients. A total of 210 patients who had undergone esophagectomy between June 1999 and March 2017 were retrospectively reviewed. AEC was defined as cancer located in the abdominal esophagus (from the hiatus to the EGJ), $>2 \mathrm{~cm}$ above the EGJ. Among all patients, 20 (9.5\%) were diagnosed with AEC. All the patients underwent preoperative endoscopy, computed tomography and esophagography to confirm the clinical diagnosis and determine the surgical approach. The right TAA was adopted for patients with cancer tissue extending $>3 \mathrm{~cm}$ above the EGJ, or with lymph node metastases (LNM) in the neck or the mediastinum.

The clinicopathological characteristics of the patients, including age, sex, histological type, TNM stage, nutritional status, use of neoadjuvant chemotherapy (NAC), surgical approach (right thoraco-abdominal or abdominal), postoperative complications, pathological findings, use of adjuvant chemotherapy and postoperative overall survival, were evaluated. The nutritional status included body mass index (BMI) and serum albumin levels. Postoperative complications included pneumonia, anastomotic leakage and surgical site infection (SSI) grade >II according to the Clavien-Dindo classification (9). The pathological findings included microscopic residual disease (R1), number of LNM and location of the LNM.

Three analyses were then performed. First, the differences in these findings were compared between SCC and adenoCa. 
Second, the differences were compared between patients who developed postoperative early recurrence (within 12 months) and patients without recurrence, to evaluate the factors associated with early recurrence. Third, a survival analysis of all AEC patients in association with the clinicopathological variables was performed using the Kaplan-Meier method and log-rank test.

Statistical analysis. The differences between the two groups were assessed using the Mann-Whitney U test for continuous variables and the Chi-squared test for categorical variables. The Kaplan-Meier method and log-rank test were used to compare the cumulative survival rates. In the survival analysis, patients were classified by the median value of the continuous variables. Statistical analysis was performed using JMP 6 software (SAS Institute Inc., Cary, NC, USA).

\section{Results}

Patient characteristics. The clinicopathological characteristics of the 20 AEC patients are summarized in Table I. The median age of the patients was 73 years and $15(75.0 \%)$ of the patients were male. A total of 11 patients $(55.0 \%)$ had SCC, $8(40.0 \%)$ had adenoCa, and $1(5.0 \%)$ had adeno SCC. Only 2 patients $(10.0 \%)$ were diagnosed as stage I, and $9(45.0 \%)$ received NAC. NAC included cisplatin and 5-fluorouracil, with or without docetaxel, as performed from 2008 onwards for clinical stage II or III patients. In 9 patients $(45.0 \%)$ the right TAA was used, while in the remaining 11 patients the abdominal approach was used. Thoracoscopy and laparoscopy were applied in 4 and 10 patients, respectively. Regarding postoperative complications, 2 patients developed pneumonia, 3 patients had anastomotic leakage, and 5 patients had SSI. As regards the pathological findings, 18 patients $(90.0 \%)$ had LNM. Only 2 patients had cervical LNM, whereas 15 (75.0\%) patients had abdominal LNM. A total of 8 patients received adjuvant chemotherapy, and recurrence occurred in 10 patients. Of those 10 patients, 4 developed early recurrence. The median survival time was 57 months. A total of 12 patients $(60.0 \%)$ had stage III disease, and their median survival time was 33 months. There was no statistically significant difference in disease-specific survival among stages $(\mathrm{P}=0.388)$.

The differences between the SCC and the adenoCa groups are summarized in Table II. Patients in the SCC group were older compared with those in the adenoCa group, although age was not significantly associated with poor disease-specific survival. The BMI and the serum albumin levels in the SCC patients were significantly lower compared with those in adenoCa patients. There were no differences in terms of surgical approach, outcome and postoperative complications. As regards pathological findings, $50 \%$ of the adenoCa group and $27.3 \%$ of the SCC group had mediastinal LNM. Abdominal LNM was more prevalent in the adenoCa group compared with the SCC group (100 vs. 54.6\%, respectively; $\mathrm{P}=0.026$ ); however, there were no significant differences in the recurrence pattern (local or distant) or in the overall survival between the two groups (Fig. 1).

A comparison between the groups with and without early recurrence is presented in Table III. The early recurrence group had a significantly longer operative time, and a greater
Table I. Characteristics of abdominal esophageal cancer patients.

\begin{tabular}{|c|c|}
\hline Characteristics & Values \\
\hline Age, years (range) & $73(52-85)$ \\
\hline Sex, male $(\%)$ & $15(75.0)$ \\
\hline \multicolumn{2}{|l|}{ Histology $(\%)$} \\
\hline Squamous cell carcinoma & $11(55.0)$ \\
\hline Adenocarcinoma & $8(40.0)$ \\
\hline Other & $1(5.0)$ \\
\hline Stage I/II/III/IV & $2 / 4 / 12 / 2$ \\
\hline BMI, kg/m² (range) & $22.0(18.1-27.2)$ \\
\hline Albumin, g/dl (range) & $3.8(3.0-4.8)$ \\
\hline NAC $(\%)$ & $9(45.0)$ \\
\hline \multicolumn{2}{|l|}{ Surgical approach (\%) } \\
\hline Thoraco-abdominal & $9(45.0)$ \\
\hline Abdominal & $11(55.0)$ \\
\hline Operative time, min (range) & $350(245-690)$ \\
\hline Blood loss, ml (range) & $305(50-2,160)$ \\
\hline \multicolumn{2}{|l|}{ Complications (\%) } \\
\hline Pneumonia & $2(10.0)$ \\
\hline Leakage & $3(15.0)$ \\
\hline Surgical site infection & $5(25.0)$ \\
\hline Hospital stay, days (range) & $21(11-198)$ \\
\hline pNO $(\%)$ & $2(10.0)$ \\
\hline Number of LNM, median (range) & $2(0-21)$ \\
\hline \multicolumn{2}{|l|}{ Location of LNM (\%) } \\
\hline Cervical LNM & $2(10.0)$ \\
\hline Mediastinal LNM & $7(35.0)$ \\
\hline Abdominal LNM & $15(75.0)$ \\
\hline Microscopic residual cancer (\%) & $4(20.0)$ \\
\hline Adjuvant therapy (\%) & $8(40.0)$ \\
\hline Recurrence (\%) & $10(50.0)$ \\
\hline Distant & $7(35.0)$ \\
\hline Local & $4(20.0)$ \\
\hline
\end{tabular}

BMI, body mass index; NAC, neo-adjuvant chemotherapy; LNM, lymph node metastasis.

prevalence of postoperative SSI and R1 resection compared with the non-early recurrence group. There was a significant difference in the overall survival between the two groups (Fig. 2).

The results of survival analysis using the Kaplan-Meier method and log-rank test is shown in Table IV. The survival time of patients who underwent TAA (Fig. 3), or had postoperative pneumonia (Fig. 4), leakage (Fig. 5), SSI (Fig. 6) and R1 (Fig. 7), was significantly shorter compared with that of patients without these factors.

\section{Discussion}

The present study demonstrated that over one-third of patients with AEC (either SCC or adenoCa) had mediastinal LNM, and postoperative complications may be associated with poor 
Table II. Comparison of the characteristics of patients with squamous cell carcinoma (SCC) and adenocarcinoma.

\begin{tabular}{|c|c|c|c|}
\hline Characteristics & $\operatorname{SCC}(n=11)$ & Adenocarcinoma $(n=8)$ & P-value \\
\hline Sex, male $(\%)$ & $9(81.8)$ & $6(75.0)$ & 0.719 \\
\hline Age, years (range) & $76(52-83)$ & $68(52-85)$ & 0.158 \\
\hline Stage I/II/III/IV & $2 / 3 / 5 / 1$ & $0 / 1 / 7 / 0$ & 0.266 \\
\hline BMI, kg/m² (range) & $21.1(18.5-22.9)$ & $24.4(20.6-27.2)$ & 0.006 \\
\hline Albumin, g/dl (range) & $3.8(3.0-4.4)$ & $4.3(3.7-4.8)$ & 0.044 \\
\hline NAC (\%) & $5(45.5)$ & $4(50.0)$ & 0.845 \\
\hline Surgical approach (\%) & & & 0.729 \\
\hline Thoraco-abdominal & $5(45.5)$ & $3(37.5)$ & \\
\hline Abdominal & $6(54.5)$ & $5(62.5)$ & \\
\hline Thoracoscopy (\%) & 2 & 2 & 1 \\
\hline Laparoscopy (\%) & $6(54.5)$ & $4(50.0)$ & 0.845 \\
\hline Operative time, min (range) & $355(245-640)$ & $313(245-690)$ & 0.836 \\
\hline Blood loss, ml (range) & $300(50-2160)$ & $260(110-580)$ & 0.620 \\
\hline \multicolumn{4}{|l|}{ Complications (\%) } \\
\hline Pneumonia & $2(18.2)$ & 0 & 0.485 \\
\hline Leakage & $3(27.3)$ & 0 & 0.107 \\
\hline Surgical site infection & $3(27.3)$ & $2(25.0)$ & 1 \\
\hline Hospital stay, days (range) & $20(11-198)$ & $26(14-35)$ & 0.710 \\
\hline pNO $(\%)$ & $2(18.2)$ & 0 & 0.477 \\
\hline Number of LNM (range) & $1.0(0-21)$ & $2.5(1-16)$ & 0.180 \\
\hline \multicolumn{4}{|l|}{ Location of LNM (\%) } \\
\hline Cervical LNM & 0 & $1(12.5)$ & 0.421 \\
\hline Mediastinal LNM & $3(27.3)$ & $4(50.0)$ & 0.377 \\
\hline Abdominal LNM & $6(54.6)$ & $8(100.0)$ & 0.026 \\
\hline Microscopic residual cancer $(\%)$ & $3(27.3)$ & $1(12.5)$ & 0.603 \\
\hline Adjuvant therapy (\%) & $2(18.2)$ & $5(62.5)$ & 0.048 \\
\hline Recurrence $(\%)$ & $5(45.5)$ & $5(62.5)$ & 0.728 \\
\hline Distant & $3(27.3)$ & $4(50.0)$ & 0.377 \\
\hline Local & $2(18.2)$ & $2(25.0)$ & 1 \\
\hline
\end{tabular}

SCC, squamous cell carcinoma; BMI, body mass index; NAC, neo-adjuvant chemotherapy; LNM, lymph node metastasis.

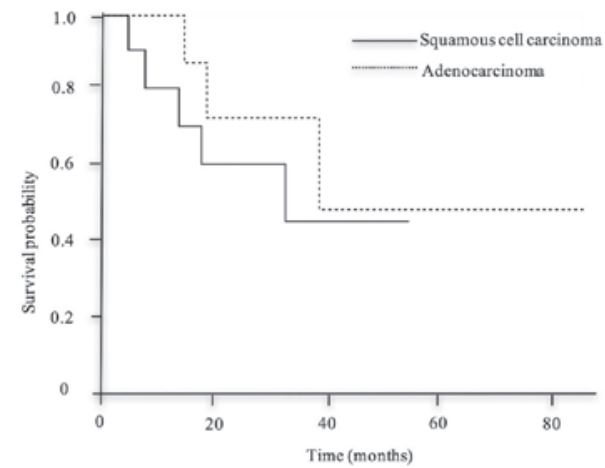

Figure 1. Kaplan-Meier estimates of postoperative disease-specific survival of patients with abdominal esophageal cancer comparing squamous cell carcinoma (solid line) and adenocarcinoma (dashed line). There was no statistically significant difference between the two groups $(\mathrm{P}=0.570)$.

prognosis. Conversely, differences in histological type and surgical approach did not appear to affect postoperative survival.
Previous studies reported that, in cases with adenoCa of the EGJ, Siewert type, histological type, tumor size and number of LNM were associated with prognosis (10). In those studies, prognosis was found to be better in Siewert type I patients compared with type II or III patients (11), and in patients with adenoCa compared with those with SCC (12). In the present study, as over half of the cohort were SCC patients, it was observed that AEC corresponded to Siewert type I, with $>30 \%$ of patients having mediastinal LNM. However, there was no difference between the SCC and adenoCa groups regarding the rate of mediastinal LNM or survival. In addition, $80 \%$ of patients who had R1 also experienced early recurrence. For these reasons, surgery for either SCC or adenoCa, without microscopic residual cancer (R0 resection), was crucial to the survival of AEC patients.

NAC or neoadjuvant chemoradiotherapy (NACRT) may achieve an increase in the $\mathrm{R} 0$ resection rate for $\mathrm{AEC}$ patients (13). However, our data do not support the efficacy of NAC and adjuvant chemotherapy. As NAC was introduced to 
Table III. Comparison of the characteristics of patients with and without early recurrence.

\begin{tabular}{|c|c|c|c|}
\hline Characteristics & Early recurrence $(n=5)$ & No early recurrence $(n=15)$ & P-value \\
\hline Sex, male $(\%)$ & $3(60.0)$ & $12(80.0)$ & 0.371 \\
\hline Age, years (range) & $72(52-81)$ & $73(52-85)$ & 0.661 \\
\hline Stage I/II/III/IV & $0 / 0 / 4 / 1$ & $2 / 4 / 8 / 1$ & 0.375 \\
\hline BMI, $\mathrm{kg} / \mathrm{m}^{2}$ (range) & $20.3(19.6-22.6)$ & $22.2(18.1-27.2)$ & 0.861 \\
\hline Albumin, g/dl (range) & $3.8(3.0-4.8)$ & $3.9(3.3-4.7)$ & 0.625 \\
\hline NAC $(\%)$ & $2(40.0)$ & $7(46.7)$ & 0.795 \\
\hline Thoraco-abdominal approach (\%) & $4(80.0)$ & $5(33.3)$ & 0.069 \\
\hline Operative time, min (range) & $630(295-690)$ & $321(245-641)$ & 0.032 \\
\hline Blood loss, ml (range) & $520(300-2,160)$ & $240(50-1,550)$ & 0.061 \\
\hline \multicolumn{4}{|l|}{ Complications (\%) } \\
\hline Pneumonia & $1(20.0)$ & $1(6.7)$ & 0.447 \\
\hline Leakage & $2(40.0)$ & $1(6.7)$ & 0.140 \\
\hline Surgical site infection & $3(60.0)$ & $2(13.3)$ & 0.037 \\
\hline Hospital stay, days (range) & $29(13-198)$ & $20(11-46)$ & 0.175 \\
\hline Number of LNM (range) & $7(1-21)$ & $2(0-8)$ & 0.024 \\
\hline \multicolumn{4}{|l|}{ Location of LNM (\%) } \\
\hline Cervical LNM & $1(20.0)$ & $1(6.7)$ & 0.447 \\
\hline Mediastinal LNM & $4(80.0)$ & $3(20.0)$ & 0.031 \\
\hline Abdominal LNM & $4(80.0)$ & $11(73.3)$ & 1 \\
\hline Microscopic residual cancer $(\%)$ & $4(80.0)$ & $0(0.0)$ & 0.001 \\
\hline Adjuvant therapy (\%) & $1(20.0)$ & $7(46.7)$ & 0.603 \\
\hline Recurrence pattern (\%) & & & 0.026 \\
\hline Local & $1(20.0)$ & $2(13.3)$ & \\
\hline Distant & $4(80.0)$ & $3(20.0)$ & \\
\hline
\end{tabular}

SCC, squamous cell carcinoma; BMI, body mass index; NAC, neo-adjuvant chemotherapy; LNM, lymph node metastasis.

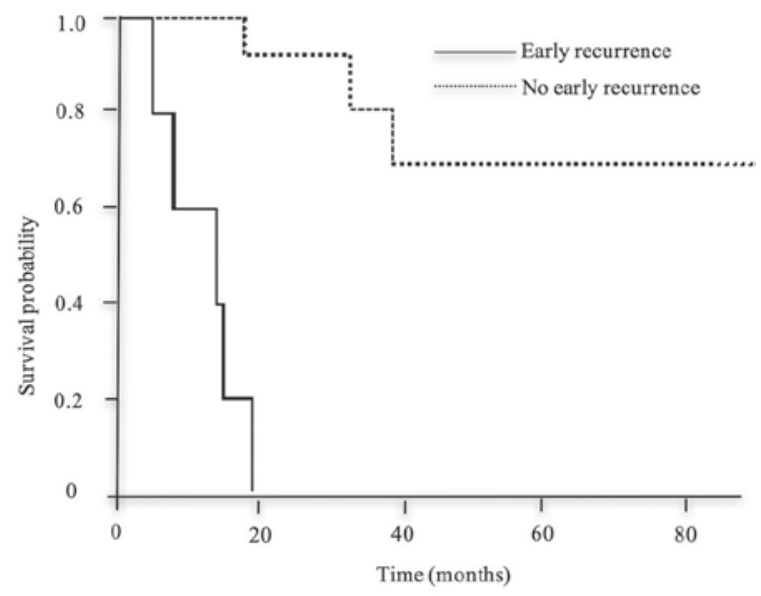

Figure 2. Kaplan-Meier estimates of postoperative disease-specific survival of patients with abdominal esophageal cancer with (solid line) and without (dashed line) early recurrence. There was a significant difference between the two groups $(\mathrm{P}<0.001)$.

treat stage II or III thoracic esophageal SCC patients in 2009, in accordance with Japanese guidelines, only 9 patients received $\mathrm{NAC}$ in the present study. A prospective study investigating the efficacy of the NAC or NACRT for AEC is required. The

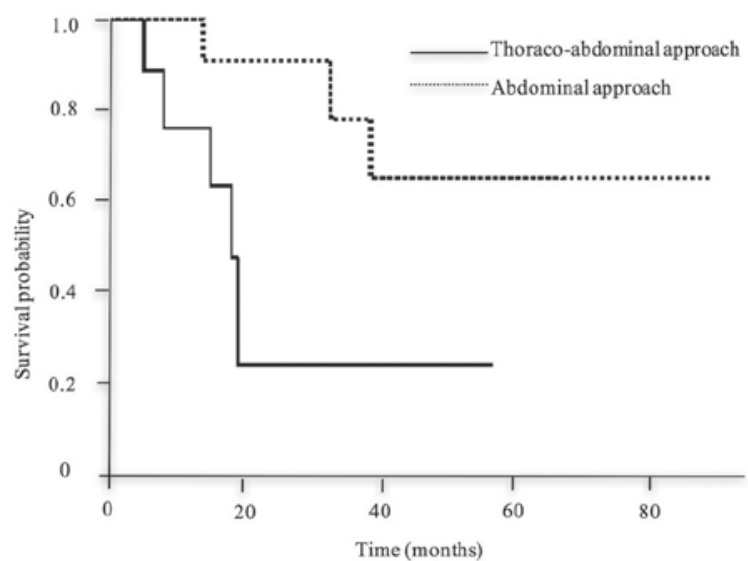

Figure 3. Kaplan-Meier estimates of postoperative disease-specific survival of patients with abdominal esophageal cancer who had undergone the thoraco-abdominal approach (solid line) or the abdominal approach (dashed line). There was a significant difference between the two groups $(\mathrm{P}=0.028)$.

survival of patients who underwent an abdominal approach was better compared with those who underwent right TAA, possibly because right TAA was performed for patients with more extensive cancer, who exhibited mediastinal or cervical LNM or thoracic esophageal invasion. Furthermore, recent 
Table IV. Evaluation of variables associated with disease-specific survival using the Kaplan-Meier method with the log-rank test.

\begin{tabular}{lccr}
\hline & \multicolumn{2}{c}{ MST, months } & \\
\cline { 2 - 3 } Variables & Yes & No & P-value \\
\hline Sex, male & NR & 33 & 0.199 \\
Age $>73$ years & 55 & 39 & 0.836 \\
BMI $>21.7 \mathrm{~kg} / \mathrm{m}^{2}$ & $\mathrm{NR}$ & 39 & 0.297 \\
Stage III or IV & NR & 33 & 0.096 \\
Neo-adjuvant chemotherapy & NR & 33 & 0.745 \\
Thoraco-abdominal approach & 18 & NR & 0.028 \\
Operative time $>345$ min & 19 & NR & 0.251 \\
Blood loss $>310$ ml & 19 & NR & 0.615 \\
Pneumonia & 18 & NR & 0.004 \\
Leakage & 8 & NR & $<0.001$ \\
Surgical site infection & 15 & NR & 0.001 \\
Microscopic residual cancer & 14 & NR & $<0.001$ \\
Number of LNM $>2$ & 19 & NR & 0.065 \\
Cervical LNM & NR & 39 & 0.888 \\
Mediastinal LNM & 19 & NR & 0.083 \\
Abdominal LNM & 39 & 48 & 0.305 \\
Adjuvant therapy & 57 & 55 & 0.540 \\
\hline
\end{tabular}

MST, median survival time; BMI, body mass index; LNM, lymph node metastasis; NR, not reached.

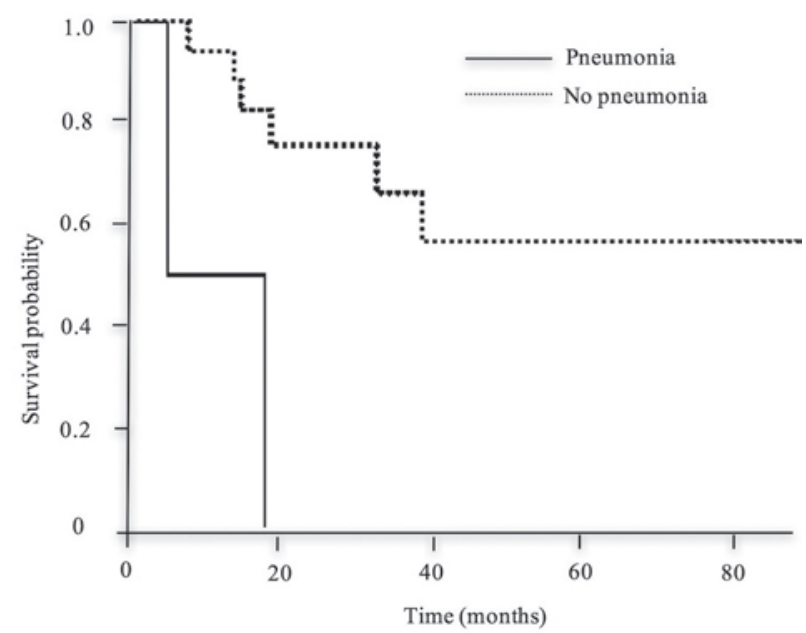

Figure 4. Kaplan-Meier estimates of postoperative disease-specific survival of patients with abdominal esophageal cancer with (solid line) or without (dashed line) postoperative pneumonia. There was a significant difference between the two groups $(\mathrm{P}=0.004)$.

advances in the use of a thoraco-laparoscopic approach for esophageal cancer may result in the development of minimally invasive surgery with $\mathrm{R} 0$ resection, which may improve the prognosis of patients compared with a conventional approach (14).

Our data suggest an association between the development of postoperative complications and a poor prognosis.

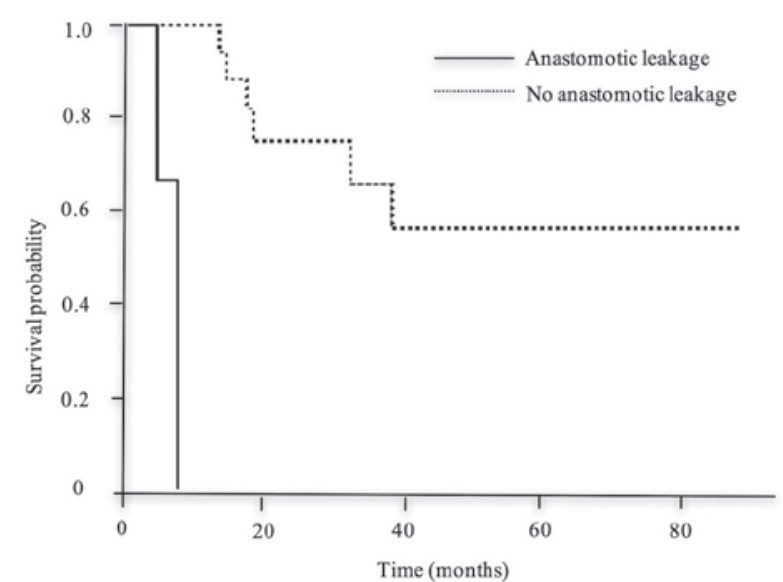

Figure 5. Kaplan-Meier estimates of postoperative disease-specific survival of patients with abdominal esophageal cancer with (solid line) or without (dashed line) postoperative anastomotic leakage. There was a significant difference between the two groups $(\mathrm{P}<0.001)$.

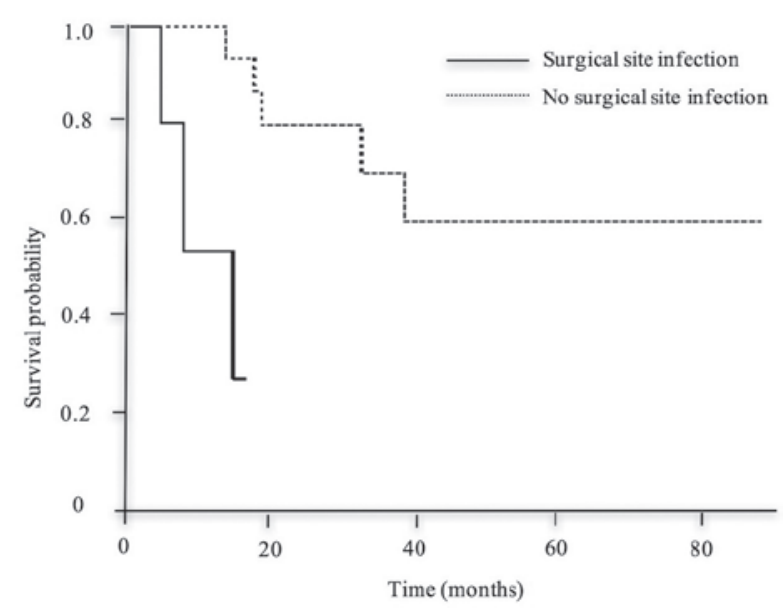

Figure 6. Kaplan-Meier estimates of postoperative overall survival of patients with abdominal esophageal cancer with (solid line) or without (dashed line) postoperative surgical site infection. There was a significant difference between the two groups $(\mathrm{P}=0.001)$.

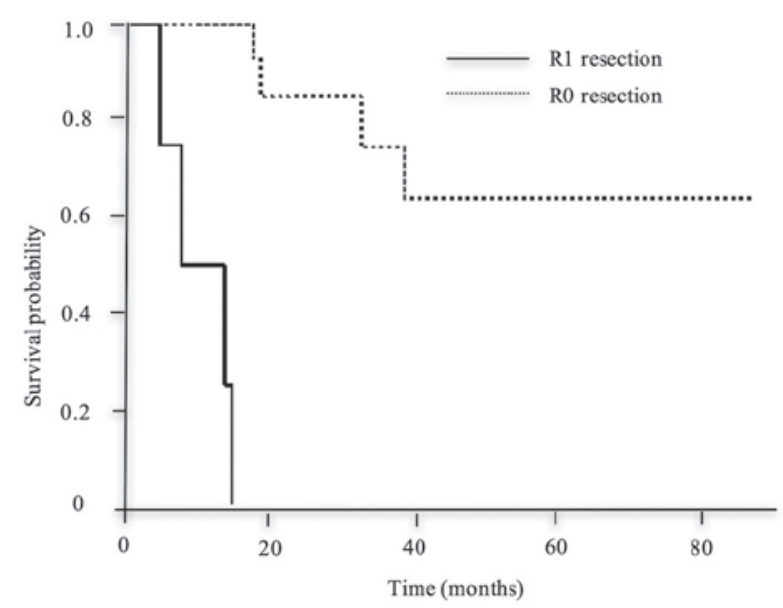

Figure 7. Kaplan-Meier estimates of postoperative overall survival of patients with abdominal esophageal cancer with microscopic residual cancer (R1 resection; solid line) or without microscopic residual cancer (R0 resection; dashed line). There was a significant difference between the two groups $(\mathrm{P}<0.001)$. 
Postoperative pneumonia after thoraco-abdominal esophagectomy has been reported to be associated with a poor prognosis $(15,16)$. Markar et al reported an association between severe anastomotic leakage and a poor prognosis from 2,944 surgically treated esophageal cancer patients (17). Although it was difficult to directly accept these data due to the high 90 -day mortality rate $(7.1 \% ; 209 / 2,944)$, these postoperative complications may suppress the patient's immunological function (18) and result in early recurrence of cancer.

The limitation of the present study is that it was retrospectively conducted with a small sample size. A multivariate analysis was performed using the Cox proportional hazards model, and found that pneumonia and R1 resection were negative predictive factors. However, due to small sample size, the hazard ratio and $95 \%$ confidence interval values were uncertain. In addition, the EGJ was difficult to define in some patients with advanced AEC, which made it more difficult to distinguish between Siewert types I and II. Further study is required to elucidate the risk of a poor prognosis for Siewert type I patients with AEC and to establish a treatment strategy.

The present study demonstrated the significance of R0 resection and prevention of postoperative complications for improving the prognosis of AEC patients. Further studies, such as prospective and multi-center investigations with large cohorts, are required to confirm short- and long-term outcomes, including the prognosis of AEC.

The present study was performed in accordance with the ethical standards laid down in the 1964 Declaration of Helsinki and its later amendments. Informed consent was obtained from all individual participants included in this study.

\section{References}

1. Wu M, Zhao JK, Hu XS, Wang PH, Qin Y, Lu YC, Yang J, Liu AM, Wu DL, Zhang ZF, et al: Association of smoking, alcohol drinking and dietary factors with esophageal cancer in high- and low-risk areas of jiangsu province, china. World J Gastroenterol 12: 1686-1693, 2006.

2. Tachimori Y, Ozawa S, Numasaki H, Fujishiro M, Matsubara $H$, Oyama T, Shinoda M, Toh Y, Udagawa H, Uno T, et al: Registration committee for esophageal cancer of the Japan esophageal society. Comprehensive registry of esophageal cancer in Japan, 2009. Esophagus 13: 110-137, 2016.

3. Ando N, Kato H, Igaki H, Shinoda M, Ozawa S, Shimizu H, Nakamura T, Yabusaki H, Aoyama N, Kurita A, et al: A randomized trial comparing postoperative adjuvant chemotherapy with cisplatin and 5-fluorouracil versus preoperative chemotherapy for localized advanced squamous cell carcinoma of the thoracic esophagus (JCOG9907). Ann Surg Oncol 19: 68-74, 2012.

4. DeMeester SR: Adenocarcinoma of the esophagus and cardia: A review of the disease and its treatment. Ann Surg Oncol 13: $12-30,2006$

5. Sjoquist KM, Burmeister BH, Smithers BM, Zalcberg JR, Simes RJ, Barbour A and Gebski V; Australasian Gastro-Intestinal Trials Group: Survival after neoadjuvant chemotherapy or chemoradiotherapy for resectable oesophageal carcinoma: An updated meta-analysis. Lancet Oncol 12: 681-692, 2011.
6. van Hagen P, Hulshof MC, van Lanschot JJ, Steyerberg EW, van Berge Henegouwen MI, Wijnhoven BP, Richel DJ, Nieuwenhuijzen GA, Hospers GA, Bonenkamp JJ, et al: Preoperative chemoradiotherapy for esophageal or junctional cancer. N Engl J Med 366: 2074-2084, 2012.

7. Siewert JR and Stein HJ: Classification of adenocarcinoma of the oesophagogastric junction. Br J Surg 85: 1457-1459, 1998.

8. Sasako M, Sano T, Yamamoto S, Sairenji M, Arai K, Kinoshita T, Nashimoto A and Hiratsuka M; Japan Clinical Oncology Group (JCOG9502): Left thoracoabdominal approach versus abdominal-transhiatal approach for gastric cancer of the cardia or subcarsia: A randomized controlled trial. Lancet Oncol 7: 644-651, 2006

9. Clavien PA, Barkun J, de Oliveira ML, Vauthey JN, Dindo D, Schulick RD, de Santibañes E, Pekolj J, Slankamenac K, Bassi C, et al: The Clavien-Dindo classification of surgical complications: Five-year experience. Ann Surg 250: 187-196, 2000.

10. Curtis NJ, Noble F, Bailey IS, Kelly JJ, Byrne JP and Underwood TJ: The relevance of the Siewert classification in the era of multimodal therapy for adenocarcinoma of the gastro-oesophageal junction. J Surg Oncol 109: 202-207, 2014.

11. Gertler R, Stein HJ, Langer R, Nettelmann M, Schuster T, Hoefler H, Siewert JR and Feith M: Long-term outcome of 2920 patients with cancers of the esophagus and esophagogastric junction: Evaluation of the new union internationale contre le cancer/american joint cancer committee staging system. Ann Surg 253: 689-698, 2011.

12. Hosokawa Y, Kinoshita T, Konishi M, Takahashi S, Gotohda N, Kato Y, Daiko H, Nishimura M, Katsumata K, Sugiyama Y and Kinoshita T: Clinicopathological features and prognostic factors of adenocarcinoma of the esophagogastric junction according to Siewert classification: Experiences at a single institution in Japan. Ann Surg Oncol 19: 677-683, 2012.

13. Sjoquist KM, Burmeister BH, Smithers BM, Zalcberg JR, Simes RJ, Barbour A and Gebski V; Australasian Gastro-Intestinal Trials Group: Survival after neoadjuvant chemotherapy or chemoradiotherapy for resectable oesophageal carcinoma: An updated meta-analysis. Lancet Oncol 12: 681-692, 2011.

14. Kitagawa $H$, Namikawa $T$, Munekage M, Fujisawa $K$, Munekgae E, Kobayashi M and Hanazaki K: Outcomes of thoracoscopic esophagectomy in prone position with laparoscopic gastric mobilization for esophageal cancer. Langenbecks Arch Surg 401: 699-705, 2016.

15. Booka E, Takeuchi H, Nishi T, Matsuda S, Kaburagi T, Fukuda K, Nakamura R, Takahashi T, Wada N, Kawakubo H, et al: The impact of postoperative complications on survivals after esophagectomy for esophageal cancer. Medicine (Baltimore) 94: e1369, 2015.

16. Kataoka K, Takeuchi H, Mizusawa J, Igaki H, Ozawa S, Abe T, Nakamura K, Kato K, Ando N and Kitagawa Y: Prognostic impact of postoperative morbidity after esophagectomy for esophageal cancer: Exploratory analysis of JCOG9907. Ann Surg 265: 1152-1157, 2017.

17. Markar S, Gronnier C, Duhamel A, Mabrut JY, Bail JP, Carrere N, Lefevre JH, Brigand C, Vaillant JC, Adham M, et al: The impact of severe anastomotic leakage on long-term survival and cancer recurrence after surgical resection for esophageal malignancy. Ann Surg 262: 972-980, 2015.

18. Okamura A, Takeuchi H, Matsuda S, Ogura M, Miyasho T, Nakamura R, Takahashi T, Wada N, Kawakubo H, Saikawa Y and Kitagawa Y: Factors affecting cytokine change after esophagectomy for esophageal cancer. Ann Surg Oncol 22: 3130-3135, 2015. 